\title{
Simulation of Fault Arc Using Conventional Arc Models
}

\author{
Ling Yuan, Lin Sun, Huaren Wu \\ School of Electrical and Automation Engineering, Nanjing Normal University, Nanjing, China \\ Email: 870599996@qq.com \\ Received April, 2013
}

\begin{abstract}
Conventional arc models are usually used to research the interaction between switching arc and circuit. It is important to simulate the fault arc for arc flash calculations, choice of electrical equipments and power system protection. This paper investigates several conventional arc models for calculating the fault arcing current. Simulation results show that conventional arc models can be used to simulate the fault arc if the parameters of arc models are given properly. This paper provides the parameters of 5 popular arc models and describes the simulation results of the fault arc.
\end{abstract}

Keywords: Arc; Arc Flash; Arc Model; Plasma; Power System

\section{Introduction}

Short circuit in power systems is serious fault. The short circuit current must be calculated for the choice of electrical equipments and the setting of power system protection. An arc presents usually at the fault location in power systems. The fault arc could destroy electrical equipment and threaten human life. It is important to calculate the arc fault current for reducing loss.

An arc model must be used for calculation of the arc fault current. There are a lot of arc models for describing the arc. Arc models can be classified in three groups: physical models, black box models and models based on graphics and diagrams. Black box models describe only the relation between input and output signals. Black box models define the interaction between the arc and the electrical circuit during the fault. In black box models, the arc is described by one differential equation or several differential equations relating the arc conductance which describes the energy balance of the arc column.

Cassie arc model was presented by Cassie in 1939 [1]. Cassie assumed that the arc has a fixed temperature being cooled by forced convection. This implies that the cross-section area of the arc is proportional to the current and that the voltage over the arc is constant. Cassie arc model is suitable for arcs with high currents.

Mayr arc model was introduced in 1943 [2, 3]. Mayr assumed that power losses are caused by thermal conduction and the arc conductance is dependent on temperature. The cross-section area of the arc is assumed constant. Mayr arc model is fit for currents near zero. Schwarz developed a modified Mayr arc model in 1971[4]. The time constant and the cooling power in the model are dependent on the arc conductance [5].
Habedank arc model is a series connection of a Cassie and Mayr arc model [6]. It is suitable for arcs with high currents and for currents near zero.

A modified Mayr arc model was presented in 1992 [7]. The cooling power in the model is current-dependent.

Schavemaker arc model is also a modified Mayr arc model with a constant time parameter [8]. The cooling power in the model is a function of the electrical power input.

The above-mentioned arc models are black box models, that is, conventional arc models. They are usually used to research the interaction between switching arc and circuit during the interruption process of circuit breakers. The characteristics of the fault arc are different from that of the switching arc. This paper describes the simulation methods of the fault arc using conventional arc models. First of all the arcing fault was tested in the high-power test laboratory and the arc voltage and the arc current are depicted. Then 5 arc models are investigated and their parameters are determined for simulation of the fault arc. Conventional arc models can be used to simulate the fault arc.

\section{Simulation of Fault Arc}

Short circuit was tested in the high-power test laboratory. The simplified laboratory test circuit is shown in Figure 1. The supply voltage is $400 \mathrm{~V}$.

The arc was initiated by means of a fuse wire between 2 electrodes. The arc voltage and the arc current were recorded with the sampling frequency of $40 \mathrm{kHz}$ and are depicted in Figure 2. Figure 2 shows that arc voltage and arc current are in phase and the arc is resistive. The arc voltage ought to be represented for simulation the 
fault arc by a periodic rectangular wave changing its sign at each zero crossing of the arc current.

The circuit equation of Figure $\mathbf{1}$ is as follows:

$$
u_{s}=R i+L \frac{d i}{d t}+u
$$

where $i$ is the arc current, $\mathrm{u}$ is the voltage across the arc and $u_{\mathrm{s}}$ is the supply voltage.

$$
u_{s}=\sqrt{2} U_{s} \sin (\omega t+\alpha)
$$

\subsection{Cassie Arc Model}

Cassie arc model represents dynamic properties of an $\mathrm{AC}$ arc by the following differential equation [1].

$$
\frac{1}{g} \frac{d g}{d t}=\frac{1}{\tau}\left(\frac{u^{2}}{U_{c}^{2}}-1\right)
$$

where $g$ is the conductance of the arc, $\tau$ is the arc time constant, $\mathrm{U}_{\mathrm{c}}$ is one constant.

$$
u=i / g
$$

$i, g$ and $u$ can be obtained by solving Equation (1), Equation (2) and Equation (3). Figure 3 shows the arc voltages. The curve 1 in Figure 3 describes the arc voltages with $\tau=0.000006$ and $\mathrm{U}_{\mathrm{c}}=80$, the curve 2 with $\tau=0.0008$ and $\mathrm{U}_{\mathrm{c}}=80$ and the curve 3 with $\tau=0.000012$ and $\mathrm{U}_{\mathrm{c}}=80$.

The curve 1 in Figure 3 shows big restrike voltage. The curve 2 is not a periodic rectangular wave. The curve 3 has the expected characteristic. The parameter $\tau$ determines the curve form and $U_{c}$ effects the mean value of the arc voltage in Figure 3. The greater $U_{c}$, the greater the mean value of the arc voltage is. Therefore, select $\tau=$ 0.000012 for simulation the fault arc and $U_{c}$ is determined by the arc voltages from test results.

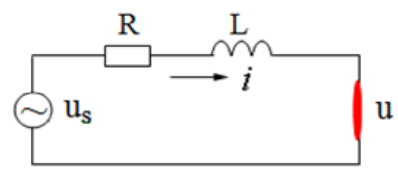

Figure 1. Test circuit.

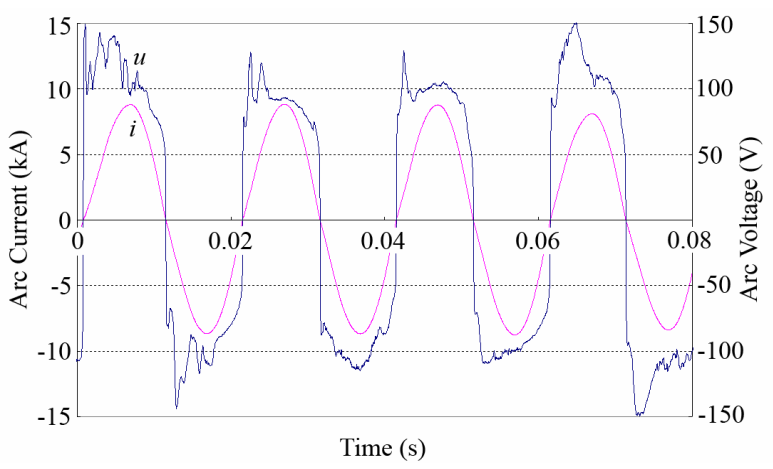

Figure 2. Test results

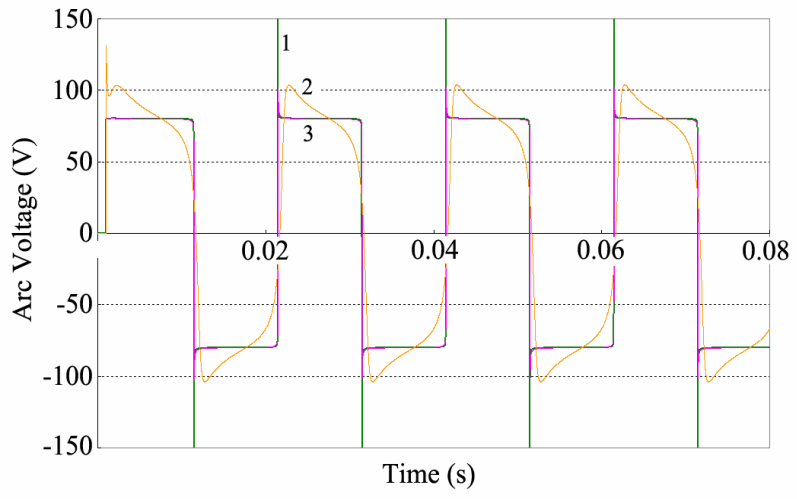

Figure 3. Arc voltages with different parameters.

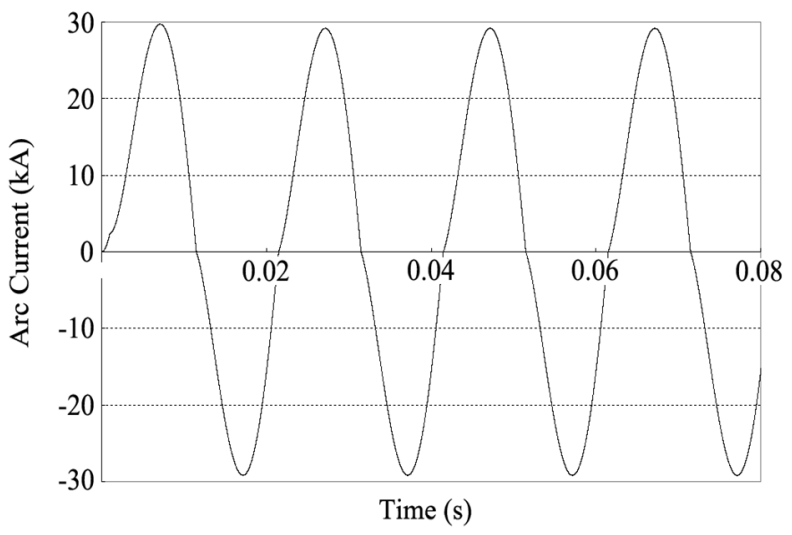

Figure 4. Arc current.

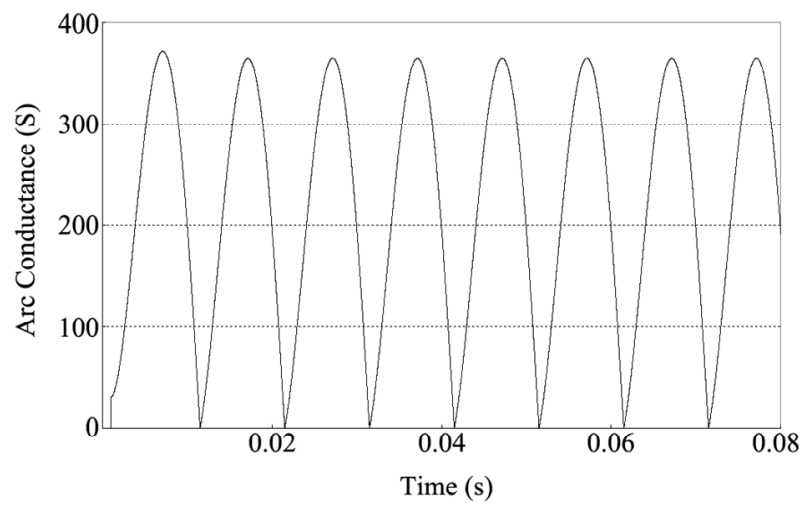

Figure 5. Arc conductance.

Figure 4 depicts an arc current with $\tau=0.000012$ and $\mathrm{Uc}=80$ and it has similar wave form as the test result in Figure 2. Figure 5 shows an arc conductance. The arc conductance is time-varying nonlinear.

Cassie arc model can also simulate three-phase short circuit arcs. A three-phase circuit is shown in Figure 6. Three-phase arcs are expressed by 3 Cassie arc models.

Figure 7 shows three-phase arc voltages. Figure 8 shows three-phase arc currents. Figure 9 shows threephase arc power. Figure 10 shows arc energy. 


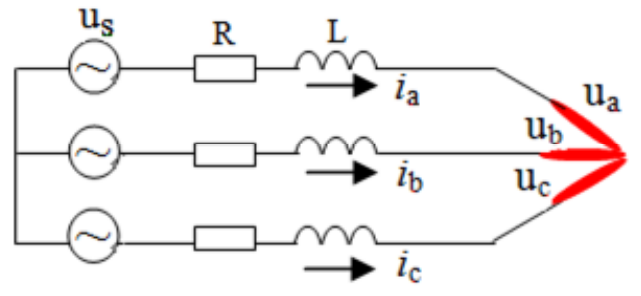

Figure 6. three-phase circuit.

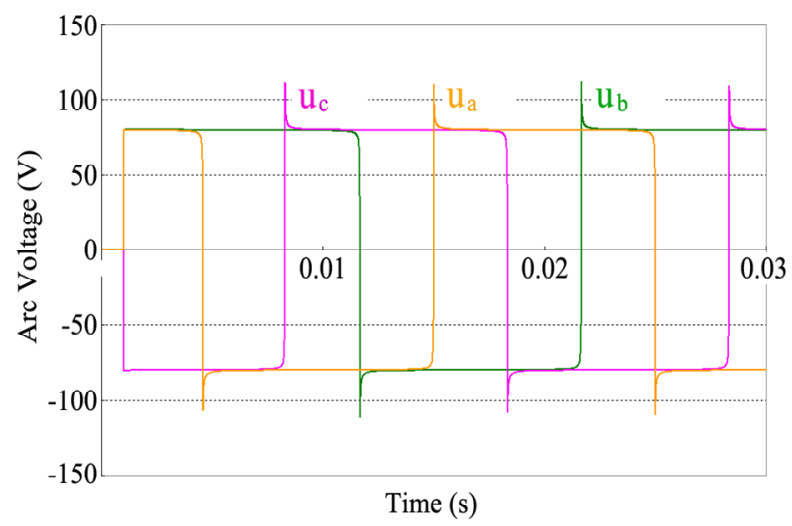

Figure 7. Three-phase arc voltages.

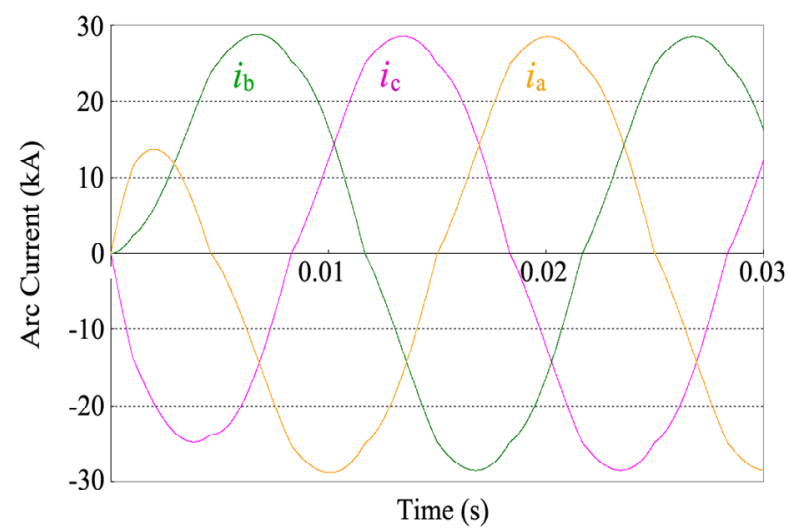

Figure 8. Three-phase arc currents.

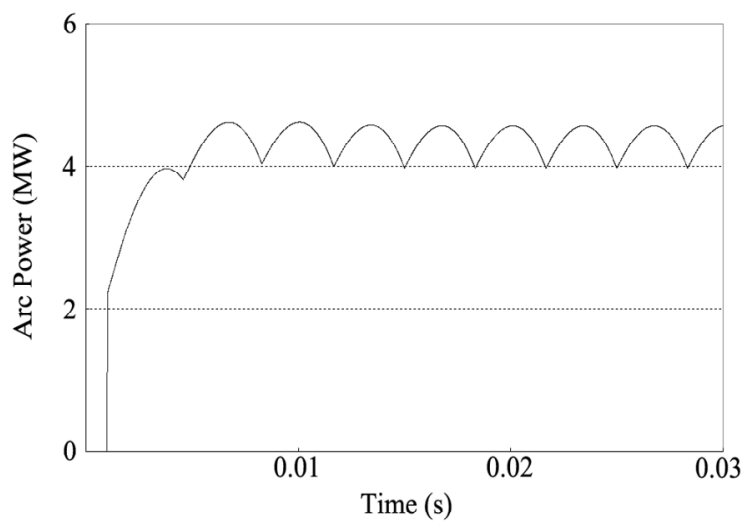

Figure 9. Three-phase arc power.

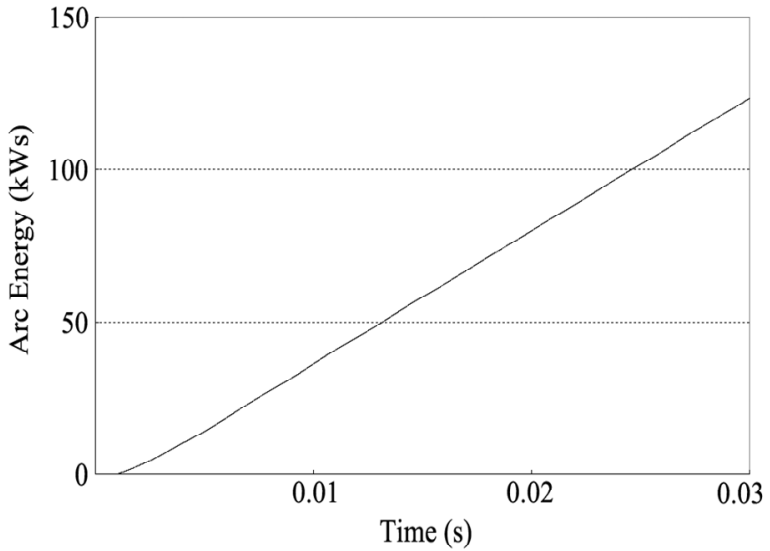

Figure 10. Three-phase arc energy.

\subsection{Schwarz Arc Model}

Schwarz arc model also includes one differential equation [4].

$$
\frac{1}{g} \frac{d g}{d t}=\frac{1}{\tau g^{a}}\left(\frac{u i}{P g^{b}}-1\right)
$$

where $\mathrm{P}$ is the cooling constant, $a$ and $b$ are the constants.

Select $\tau=0.0001, \mathrm{P}=2000, \mathrm{a}=0.1$ and $\mathrm{b}=1.2$. $i, g$ and $u$ are obtained by solving Equation (1), Equation (3) and Equation (4). The arc voltage is shown in Figure 11 and the arc current in Figure 12. The curve of the arc voltage in Figure $\mathbf{1 1}$ has expected form. The parameters $\tau, a$ and $b$ define the waveform of the arc voltage, and $\mathrm{P}$ ensures the mean value of the arc voltage. So $\tau=0.0001, a=0.1$ and $b=1.2$ are used and the parameter $\mathrm{P}$ is determined by the arc voltages from test results.

\subsection{Habedank Arc Model}

Habedank arc model is a series connection of a Cassie and Mayr arc model and consists of two differential equations and one algebraic equation [6].

$$
\begin{aligned}
\frac{1}{g_{c}} \frac{d g_{c}}{d t} & =\frac{1}{\tau_{c}}\left(\left(\frac{u g}{U_{c} g_{c}}\right)^{2}-1\right) \\
\frac{1}{g_{m}} \frac{d g_{m}}{d t} & =\frac{1}{\tau_{m}}\left(\frac{u^{2} g^{2}}{P_{0} g_{m}}-1\right) \\
\frac{1}{g} & =\frac{1}{g_{c}}+\frac{1}{g_{m}}
\end{aligned}
$$

where $g_{c}$ is the arc conductance in the Cassie equation, $\tau_{c}$ is the Cassie time constant, $g_{m}$ is the arc conductance in the Mayr equation, $\tau_{\mathrm{m}}$ is the Mayr time constant and $\mathrm{P}_{0}$ is the constant.

Solve Equation (1), Equation (3), Equation (5), Equation (6) and Equation (7) to get $i, g$ and $u$. The arc volt- 
age is shown in Figure $\mathbf{1 3}$ and the arc current in Figure 14 with $\tau_{\mathrm{c}}=0.0001, \tau_{\mathrm{m}}=0.0001, \mathrm{P}_{0}=100$ and $\mathrm{U}_{\mathrm{c}}=80$. The curve of the arc voltage in Figure $\mathbf{1 3}$ has expected form and Habedank arc model can simulate the fault arc. Therefore select $\tau_{\mathrm{c}}=0.0001, \tau_{\mathrm{m}}=0.0001$ and $\mathrm{P}_{0}=100$, and $\mathrm{U}_{\mathrm{c}}$ is determined by the arc voltages from test results.

\subsection{Modified Mayr Arc Model}

Modified Mayr arc model contains one differential equation [7].

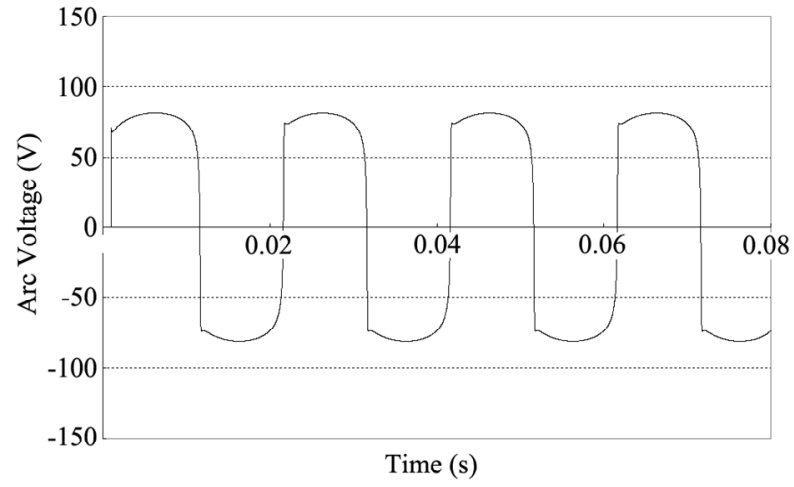

Figure 11. Arc voltage simulated by Schwarz arc model.

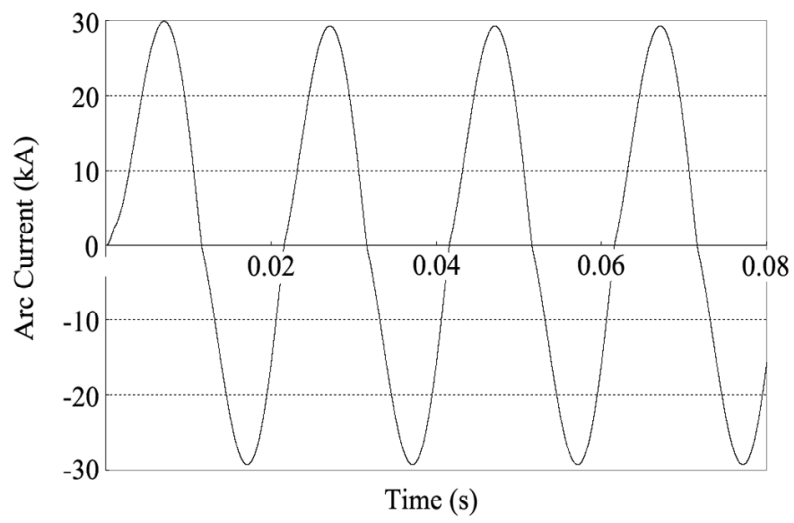

Figure 12. Arc current simulated by Schwarz arc model.

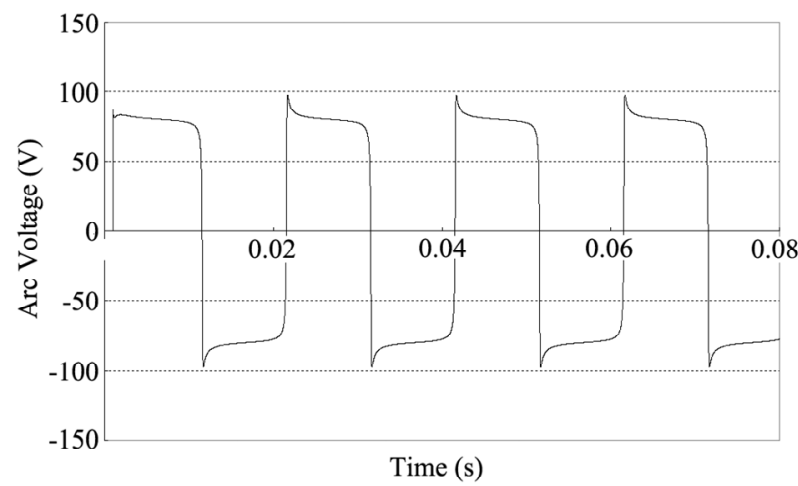

Figure 13. Arc voltage simulated by Habedank arc model.

$$
\frac{1}{g} \frac{d g}{d t}=\frac{1}{\tau}\left(\frac{u i}{P_{0}+C_{i}|i|}-1\right)
$$

where $C_{i}$ is the current constant.

The arc voltage is shown in Figure $\mathbf{1 5}$ and the arc current in Figure 16 with $\tau=0.000012, \mathrm{P}_{0}=1000$ and $C_{i}=$ 80. The curve of the arc voltage in Figure 15 has expected waveform and Modified Mayr arc model can simulate the fault arc. Therefore select $\tau_{\mathrm{c}}=0.000012$, and $\mathrm{P}_{0}=1000$, and $C_{i}$ is determined by the arc voltages from test results.

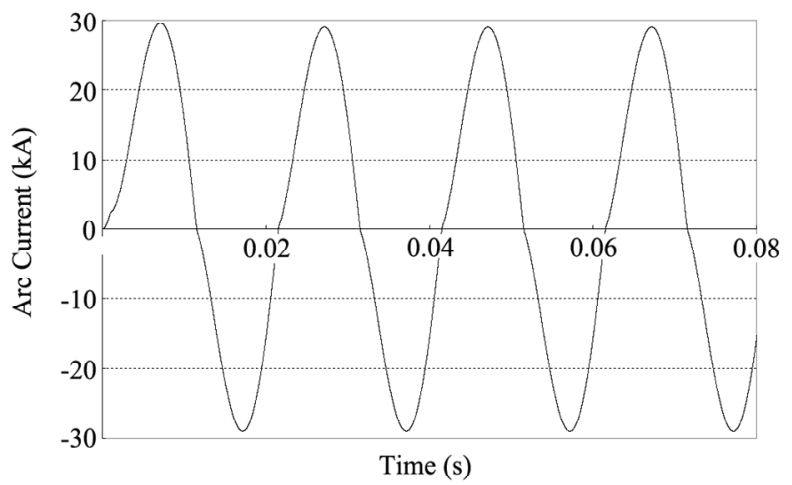

Figure 14. Arc current simulated by Habedank arc model.

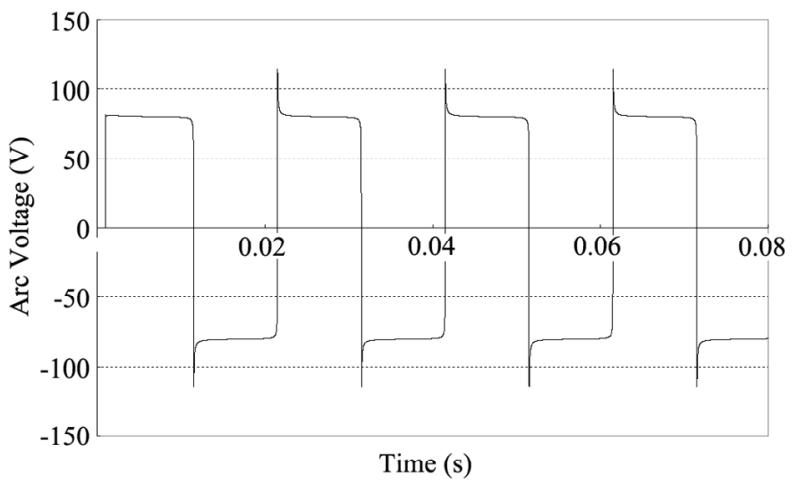

Figure 15. Arc voltage simulated by modified Mayr model.

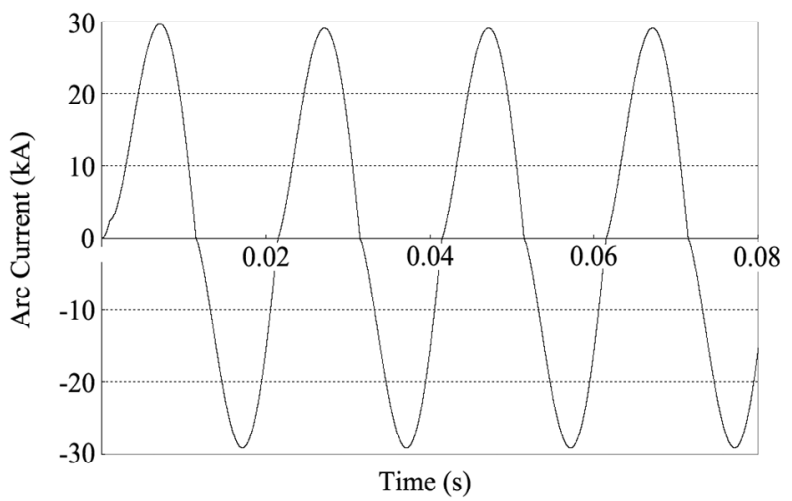

Figure 16. Arc current simulated by modified Mayr model. 


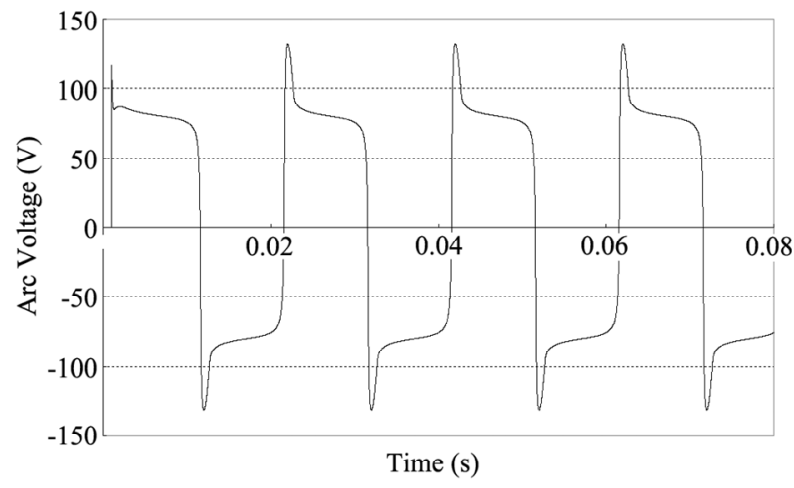

Figure 17. Arc voltage simulated by Schavemaker model.

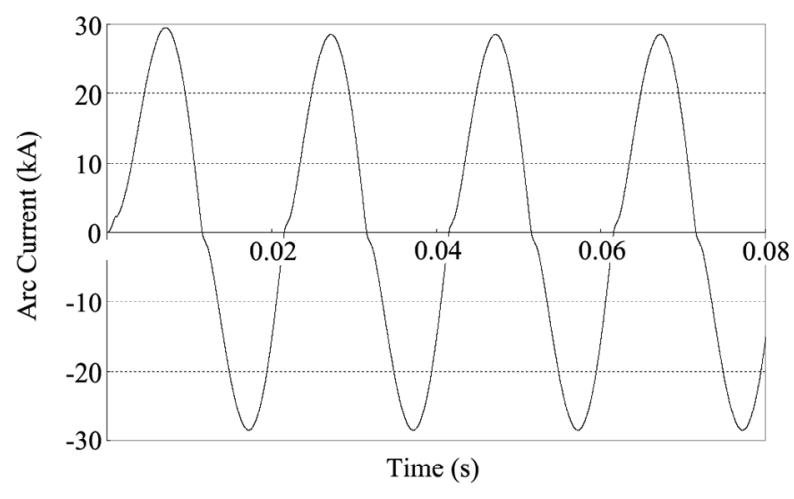

Figure 18. Arc current simulated by Schavemaker model.

\subsection{Schavemaker Arc Model}

Schavemaker arc model is a modified Mayr arc model with a constant time parameter $\tau$ and the cooling power that is a function of the electrical power input. Schavemaker arc model includes one differential equation [8].

$$
\frac{1}{g} \frac{d g}{d t}=\frac{1}{\tau}\left(\frac{u i}{\max \left(U_{\text {arc }}|i|, P_{0}+P_{1} u i\right)}-1\right)
$$

where $P_{1}$ is the cooling constant and $U_{\text {arc }}$ is the constant that determines the mean value of the arc voltage.

The arc voltage is shown in Figure $\mathbf{1 7}$ and the arc current in Figure 18 with $\tau=0.0001, \mathrm{P}_{0}=10000, \mathrm{P}_{1}=0.8$ and $\mathrm{U}_{\text {arc }}=80$. Consequently, $\tau=0.0001, \mathrm{P}_{0}=10000$ and $\mathrm{P}_{1}=0.8$ are used and $\mathrm{U}_{\text {arc }}$ depends on the arc voltages from test results for simulation of the fault arc.

\section{Conclusions}

The fault arc could destroy electrical equipment and threaten human life. It is important to research the simu- lation of the fault arc. Black box models are simple and suitable to simulate the fault arc for arc flash calculations, choice of electrical equipments and power system protection. The arc voltage ought to be represented by a periodic rectangular wave changing its sign at each zero crossing of the arc current for simulation the fault arc. Some black box models can create the arc voltage with the expected waveform if the parameters of arc model are given properly. This paper provides the parameters of 5 popular arc models and describes the simulation results of the fault arc. Schwarz arc model is the best arc model of 5 arc models for the simulation the fault arc.

This paper investigated conventional arc models for the arcing fault with fixed electrodes. The next task will be to research conventional arc models for the simulation of the arcing fault occurred at overhead lines.

\section{REFERENCES}

[1] A. M. Cassie, "Theorie Nouvelle des Arcs de Rupture et de la Rigidité des Circuits," CIGRE, Vol. 102, 1939, pp. 588-608.

[2] O. Mayr, "Beitrage zur Theorie des Statischen und des Dynamischen Lichtbogens," Archiv für Elektrotechnik, Vol. 37, No. 12, 1943, pp. 588-608.

[3] O. Mayr, "Über die Theorie des Lichtbogens und seiner Löschung," Elektrotechnische Zeitschrift, Jahrgang, Vol. 64, No. 49-50, 1943, pp. 645-652.

[4] J. Schwarz, "Dynamisches Verhalten eines Gasbe- blasenen, Turbulenzbestimmten Schaltlichtbogens," ETZ-A, Vol. 92, 1971, pp. 389-391.

[5] P. H. Schavemaker, "Arc Model Blockset for use with MATLAB Simulink and Power System: Blockset-User's Guide,"

http://www.ewi.tudelft.nl/fileadmin/Faculteit/EWI/Over de_faculteit/Afdelingen/Electrical_Sustainable_Energy/ $\overline{\mathrm{H}}$ CPS/EPS/downloads/doc/amb2.zip

[6] U. Habedank, "On the Mathematical Description of Arc Behaviour in the Vicinity of Current Zero," etzArchiv, Vol. 10, No.11, 1988, pp. 339-343.

[7] L. Van der Sluis, W. R. Rutgers and C. G. A. Koreman, "A Physical Arc Model for the Simulation of Current Zero Behaviour of High-Voltage Circuit Breakers," IEEE Transactions on Power Delivery, Vol. 7, No. 2, 1992, pp. 1016-1022. doi:10.1109/61.127112

[8] P. H. Schavemaker and L. Van der Sluis, "An Improved Mayr-Type Arc Model Based on Current-Zero Measurements," IEEE Transactions on Power Delivery, Vol. 15, No. 2, 2000, pp. 580-584.doi:10.1109/61.852988 